\title{
Descriptive comparison of the rating scales through different scale estimates. Simulation-based analysis
}

\author{
Irene Arellano ${ }^{1}$, Beatriz Sinova ${ }^{1}$, Sara de la Rosa de Sáa ${ }^{2}$, \\ María Asunción Lubiano ${ }^{1}$, and María Ángeles Gil ${ }^{1}$ \\ 1 Departamento de Estadística, I.O. y D.M., Universidad de Oviedo, \\ C/ Federico García Lorca 18, 33007 Oviedo, Spain, \\ uo239511@uniovi.es, sinovabeatriz@uniovi.es, lubiano@uniovi.es, \\ magil@uniovi.es, \\ 2 Oficina de Evaluación de Tecnologías Sanitarias, Servicio de Salud del Principado \\ de Asturias, Asturias, Spain, \\ sara.delarosa@sespa.es
}

\begin{abstract}
In dealing with intrinsically imprecise-valued magnitudes, a common rating scale type is the natural language-based Likert. Along the last decades, fuzzy scales (more concretely, fuzzy linguistic scales/variables and fuzzy ratig scales) have also been considered for rating values of these magnitudes. A comparative descriptive analysis focussed on the variability/dispersion associated with the magnitude depending on the considered rating scale is performed in this study. Fuzzy rating responses are simulated and associated with Likert responses by means of a 'Likertization' criterion. Then, each 'Likertized' datum is encoded by means of a fuzzy linguistic scale. In this way, with the responses available in the three scales, the value of the different dispersion estimators is calculated and compared among the scales.
\end{abstract}

Keywords: fuzzy linguistic scale, fuzzy rating scale, Likert scale, scale estimates

\section{Introduction}

The Likert-type scales are frequently used in designing questionnaires to rate characteristics or attributes that cannot be numerically measured (like satisfaction, perceived quality, perception...). Although they are easy to answer and they do not require a special training to use them, respondents often do not find accurate answers to items and the available statistical methodology to analyze the data from these questionnaires is rather limited. This is mainly due to the fact that Likert scales are discrete with a very small number of responses to choose for each item (often 4 to 7). To overcome this concern, Hesketh et $a l$. [5] proposed the so-called fuzzy rating scale to allow a complete freedom and expressiveness in responding, without respondents being constrained to choose among a few pre-specified responses. 
By drawing the fuzzy number that best represents the respondent's valuation, the fuzzy rating scale captures the logical imprecision associated with such variables. Moreover, this fuzzy rating scale allows us to have a rich continuous scale of measurement, unlike the case of a posterior numerical or fuzzy encoding (the latter encoding Likert points with fuzzy numbers from a linguistic scale, and usually made by trained experts).

In previous studies (see Gil et al. [3], Lubiano et al. [7,6,8]) we have confirmed that the results when fuzzy rating scales are considered sometimes differ importantly from the conclusions drawn from numerically or fuzzy linguistically encoded Likert values.

As differences can often be even clearer from the dispersion than for the location perspective, this paper aims to examine, by means of simulation developments, how location-based 'scale' estimates are affected by the considered scale of measurement.

\section{Preliminaries}

A (bounded) fuzzy number is a mapping $\widetilde{U}: \mathbb{R} \rightarrow[0,1]$ such that for all $\alpha \in[0,1]$, the $\alpha$-level set $\widetilde{U}_{\alpha}=\{x \in \mathbb{R}: \widetilde{U}(x) \geq \alpha\}$ if $\alpha \in(0,1]$, and $\widetilde{U}_{0}=$ $\operatorname{cl}\{x \in \mathbb{R}: \widetilde{U}(x)>0\}$ (with 'cl' denoting the closure of the set) is a nonempty compact interval.

In dealing with fuzzy number-valued data, distances will be computed by considering two different metrics introduced by Diamond and Kloeden [1]: the 2-norm metric $\rho_{2}$ and the 1-norm metric $\rho_{1}$, which for fuzzy numbers $\widetilde{U}$ and $\widetilde{V}$ are given by

$$
\begin{gathered}
\rho_{2}(\widetilde{U}, \widetilde{V})=\sqrt{\frac{1}{2} \int_{[0,1]}\left[\left(\inf \widetilde{U}_{\alpha}-\inf \widetilde{V}_{\alpha}\right)^{2}+\left(\sup \widetilde{U}_{\alpha}-\sup \widetilde{V}_{\alpha}\right)^{2}\right] d \alpha} \\
\rho_{1}(\widetilde{U}, \widetilde{V})=\frac{1}{2} \int_{[0,1]}\left[\left|\inf \widetilde{U}_{\alpha}-\inf \widetilde{V}_{\alpha}\right|+\left|\sup \widetilde{U}_{\alpha}-\sup \widetilde{V}_{\alpha}\right|\right] d \alpha .
\end{gathered}
$$

\section{Scales measures for fuzzy data}

In developing statistics with fuzzy data coming from intrinsically imprecisevalued attributes, random fuzzy numbers constitute a well-formalized model within the probabilistic setting for the random mechanisms generating such data.

Let $\mathcal{X}$ be a random fuzzy number (as defined by Puri and Ralescu [9]) associated with a probability space, i.e., a fuzzy number-valued mapping $\mathcal{X}$ associated with a probability space and such that, for each $\alpha$, the $\alpha$-level interval-valued mapping is a random interval associated with the probability space.

Let $\widetilde{\mathbf{x}}_{n}=\left(\widetilde{x}_{1}, \ldots, \widetilde{x}_{n}\right)$ be a sample of observations from $\mathcal{X}$. The sample Aumann-type mean is the fuzzy number such that for each $\alpha$ 


$$
\left(\overline{\widetilde{\mathbf{x}}}_{n}\right)_{\alpha}=\left[\sum_{i=1}^{n} \inf \left(\widetilde{x}_{i}\right)_{\alpha} / n, \sum_{i=1}^{n} \sup \left(\widetilde{x}_{i}\right)_{\alpha} / n\right],
$$

and the sample 1-norm median is the fuzzy number such that for each $\alpha$

$$
\left(\widehat{\overline{\mathrm{Me}}}\left(\widetilde{\mathbf{x}}_{n}\right)\right)_{\alpha}=\left[\mathrm{Me}_{i} \inf \left(\widetilde{x}_{i}\right)_{\alpha}, \mathrm{Me}_{i} \sup \left(\widetilde{x}_{i}\right)_{\alpha}\right] .
$$

In De la Rosa de Sáa et al. [2] one can find together the most commonly used location-based scale estimates, namely: the sample Fréchet-type $\rho_{\mathbf{2}}$-Standard Deviation and, for $D \in\left\{\rho_{1}, \rho_{2}\right\}$ and $\widetilde{M} \in\left\{\overline{\widetilde{\mathbf{x}}}_{n}, \widehat{\widetilde{M}}\left(\widetilde{\mathbf{x}}_{n}\right)\right\}$, the sample $D$ Average Distance Deviation and the sample D-Median Distance Deviation, which are respectively given by

$$
\begin{gathered}
\rho_{2}-\mathrm{SD}\left(\widetilde{\mathbf{x}}_{n}\right)=\sqrt{\frac{1}{n} \sum_{i=1}^{n}\left[\rho_{2}\left(\widetilde{x}_{i}, \overline{\mathbf{x}}_{n}\right)\right]^{2}} \\
\widehat{D-\mathrm{ADD}}\left(\widetilde{\mathbf{x}}_{n}, \widetilde{M}\right)=\frac{1}{n} \sum_{i=1}^{n} D\left(\widetilde{x}_{i}, \widetilde{M}\right), \widehat{D-\operatorname{MDD}}\left(\widetilde{\mathbf{x}}_{n}, \widetilde{M}\right)=\operatorname{Me}_{i}\left\{D\left(\widetilde{x}_{i}, \widetilde{M}\right)\right\} .
\end{gathered}
$$

\section{Generating fuzzy data for simulation studies}

In this work, simulations have been inspired by real-life datasets in connection with fuzzy rating scale-based experiments.

To generate fuzzy data from a trapezoidal-valued random fuzzy number $\mathcal{X}=\operatorname{Tra}\left(\inf \mathcal{X}_{0}, \inf \mathcal{X}_{1}, \sup \mathcal{X}_{1}, \sup \mathcal{X}_{0}\right)$, Sinova et al. [10] suggest to use an alternative characterization, $\mathcal{X}=\operatorname{Tra}\left\langle X_{1}, X_{2}, X_{3}, X_{4}\right\rangle$, where (see Figure 1)

$$
\begin{gathered}
X_{1}=\operatorname{mid} \mathcal{X}_{1}=\left(\inf \mathcal{X}_{1}+\sup \mathcal{X}_{1}\right) / 2, \quad X_{2}=\operatorname{spr} \mathcal{X}_{1}=\left(\sup \mathcal{X}_{1}-\inf \mathcal{X}_{1}\right) / 2, \\
X_{3}=\operatorname{lspr} \mathcal{X}_{0}=\inf \mathcal{X}_{1}-\inf \mathcal{X}_{0}, \quad X_{4}=\text { uspr } \mathcal{X}_{0}=\sup \mathcal{X}_{0}-\sup \mathcal{X}_{1},
\end{gathered}
$$

(i.e., $X_{1}=$ core mid-point, $X_{2}=$ core radius, $X_{3}=$ 'left distance' between core and support, $X_{4}=$ 'right distance' between core and support) whence

$\mathcal{X}=\operatorname{Tra}\left\langle X_{1}, X_{2}, X_{3}, X_{4}\right\rangle=\operatorname{Tra}\left(X_{1}-X_{2}-X_{3}, X_{1}-X_{2}, X_{1}+X_{2}, X_{1}+X_{2}+X_{4}\right)$.

In fact, fuzzy data will be generated by simulating the four real-valued random variables $X_{1}, X_{2}, X_{3}$ and $X_{4}$, so that the $\mathbb{R} \times[0, \infty) \times[0, \infty) \times[0, \infty)$-valued random vector $\left(X_{1}, X_{2}, X_{3}, X_{4}\right)$ will provide us with the 4 -tuples $\left(x_{1}, x_{2}, x_{3}, x_{4}\right)$ with $x_{1}=$ center and $x_{2}=$ radius of the core, and $x_{3}=$ lower and $x_{4}=$ upper spread of the fuzzy number. To each generated 4 -tuple $\left(x_{1}, x_{2}, x_{3}, x_{4}\right)$ we associate the fuzzy number $\operatorname{Tra}\left\langle x_{1}, x_{2}, x_{3}, x_{4}\right\rangle$.

According to the simulation procedure, data have been generated from random fuzzy numbers with a bounded reference set and abstracting and mimicking what we have observed in real-life examples employing the fuzzy rating scale (FRS). More concretely, fuzzy data have been generated such that 


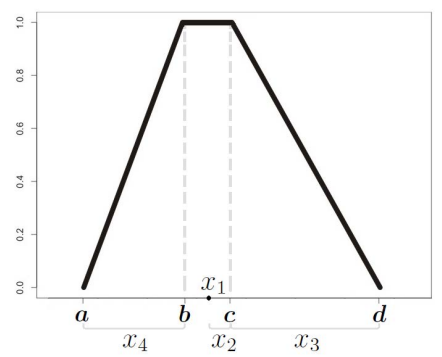

Fig. 1. 4-Tuples to be generated for the simulation procedures

- $100 \cdot \omega_{1} \%$ of the data have been obtained by first considering a simulation from a simple random sample of size 4 from a beta $\beta(p, q)$ distribution, ordering the corresponding 4 -tuple, and finally computing the values $x_{i}$. The values of $p$ and $q$ vary in most cases to cover different distributions (namely, symmetrical weighting central values, symmetrical weighting extreme values, and asymmetric ones). In most of the comparative studies involving simulations, the values from the beta distribution are re-scaled and translated to an interval $\left[l_{0}, u_{0}\right]$ different from $[0,1]$.

- $100 \cdot \omega_{2} \%$ of the data have been obtained considering a simulation of four random variables $X_{i}=\left(u_{0}-l_{0}\right) \cdot Y_{i}+l_{0}$ as follows:

$$
\begin{aligned}
Y_{1} & \sim \beta(p, q), \\
Y_{2} & \sim \text { Uniform }\left[0, \min \left\{1 / 10, Y_{1}, 1-Y_{1}\right\}\right], \\
Y_{3} & \sim \text { Uniform }\left[0, \min \left\{1 / 5, Y_{1}-Y_{2}\right\}\right], \\
Y_{4} & \sim \text { Uniform }\left[0, \min \left\{1 / 5,1-Y_{1}-Y_{2}\right\}\right] .
\end{aligned}
$$

- $100 \cdot \omega_{3} \%$ of the data have been obtained considering a simulation of four random variables $X_{i}=\left(u_{0}-l_{0}\right) \cdot Y_{i}+l_{0}$ as follows:

$$
\begin{aligned}
Y_{1} & \sim \beta(p, q), \\
Y_{2} & \sim \begin{cases}\operatorname{Exp}(200) & \text { if } Y_{1} \in[0.25,0.75] \\
\operatorname{Exp}\left(100+4 Y_{1}\right) & \text { if } Y_{1}<0.25 \\
\operatorname{Exp}\left(500-4 Y_{1}\right) & \text { otherwise }\end{cases} \\
Y_{3} & \sim \begin{cases}\gamma(4,100) & \text { if } Y_{1}-Y_{2} \geq 0.25 \\
\gamma\left(4,100+4 Y_{1}\right) & \text { otherwise }\end{cases} \\
Y_{4} & \sim \begin{cases}\gamma(4,100) & \text { if } Y_{1}+Y_{2} \geq 0.25 \\
\gamma\left(4,500-4 Y_{1}\right) & \text { otherwise. }\end{cases}
\end{aligned}
$$

\section{$5 \quad$ Results}

First, FRS data will be simulated in accordance with the above described realistic simulation procedure. Later, fuzzy data based on a fuzzy rating scale can fairly be associated/classified in accordance with labels in a Likert scale (more concretely, with their numerical encoding). This process is to be called "Likertization". Furthermore, the associated Likert values could also be later encoded by means of values from a fuzzy linguistic scale. 
For carrying out the Likertization, the "minimum distance Likertization criterion" will be employed (see Figure 2):

FRS-based response

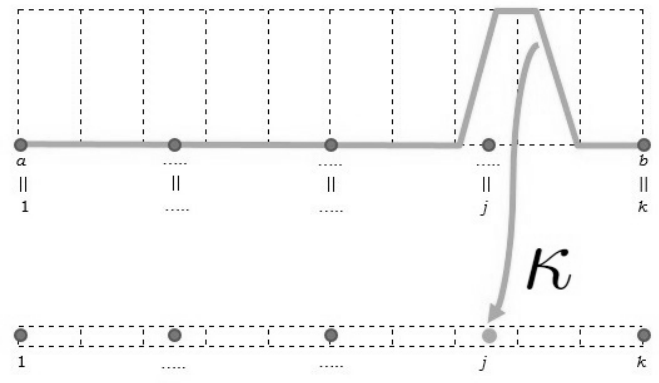

$\boldsymbol{k}$-point $\kappa$-associated response

Fig. 2. Minimum distance criterion scheme when the reference interval equals $[1, k]$

In this way, if the considered Likert scale is a $k$-point one, given a metric $D$ between fuzzy data and $\widetilde{U}$ the free fuzzy response to be classified, then $\widetilde{U}$ is associated with the integer $\kappa(\widetilde{U})$ such that

$$
\kappa(\widetilde{U})=\arg \min _{j \in\{1, \ldots, k\}} D\left(\widetilde{U}, \mathbb{1}_{\{j\}}\right) .
$$

Each FRS-based datum will be first Likertized by means of the minimum distance criterion, and it will later be encoded by means of a fuzzy linguistic scale. We have chosen the most usual (see, for instance, Herrera et al. [4]) balanced semantic representations of the linguistic hierarchies of $k=4$ and $k=5$ levels (Figure 3).
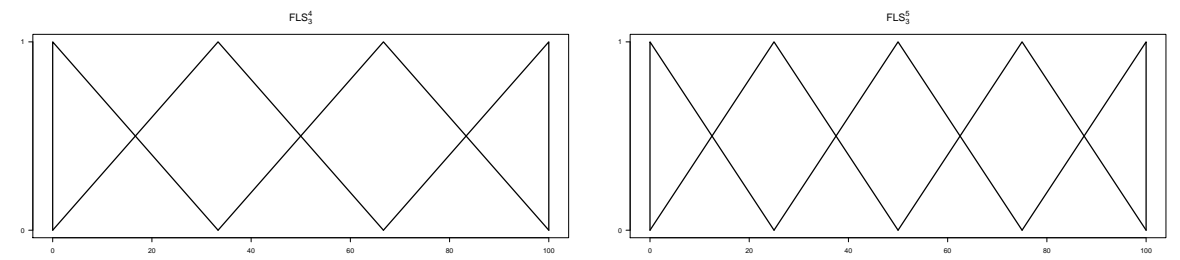

Fig. 3. Usual balanced semantic representation of the linguistic hierarchies of $k=4$ and $k=5$ levels

Simulations-based tables (Tables 1 and 2) collect the percentages of Euclidean distances between the sample scale estimates $\widehat{D}$ for the FRS-simulated data and for their numerically (NEL) and fuzzy linguistically (FLS) encoded $\rho_{1}$ Likertization that are over $\varepsilon \in\{1,5,10,15\}$. 
Table 1. \% of simulated samples of size $n$ for which the Euclidean distance between the sample scale estimate $\widehat{D}$ associated with the FRS and the one associated with either the NEL (numerically encoded Likert) or the FLS (fuzzy linguistic scale) with $k=4$ different values is greater than $\varepsilon \in\{1,5,10,15\}$ and (from top to bottom) $\beta(p, q) \equiv \beta(1,1), \beta(.75, .75), \beta(4,2)$, and $\beta(6,1)$

\begin{tabular}{|c|c|c|c|c|c|c|c|c|c|}
\hline \multirow{3}{*}{$\frac{\widehat{\mathrm{D}}}{\widehat{\rho_{2-\mathrm{SD}}}\left(\widetilde{\mathbf{x}}_{n}\right)}$} & \multirow{3}{*}{$\begin{array}{c}n \\
10 \\
30 \\
100\end{array}$} & \multicolumn{8}{|c|}{$\%|\hat{\mathrm{D}}(\mathrm{FRS})-\hat{\mathrm{D}}(\mathrm{S})|>\varepsilon \quad(k=4, \beta(p, q) \equiv \beta(1,1))$} \\
\hline & & \multicolumn{2}{|c|}{$\mathrm{S}=\mathrm{NEL}=1$} & \multicolumn{2}{|c|}{$\mathrm{S}=\mathrm{NEL} \mid \mathrm{S}=\mathrm{FLS}$} & \multicolumn{2}{|c|}{$\mathrm{S}=\mathrm{NEL}=10=\mathrm{FLS}$} & \multicolumn{2}{|c|}{$\mathrm{S}=\mathrm{NEL} \mid \mathrm{S}=\mathrm{FLS}$} \\
\hline & & $\begin{array}{l}82.2 \\
85.7 \\
94.9\end{array}$ & $\begin{array}{l}64.3 \\
51.9 \\
44.2\end{array}$ & $\begin{array}{c}16.8 \\
4.8 \\
0\end{array}$ & $\begin{array}{c}4.1 \\
0.1 \\
0\end{array}$ & $\begin{array}{c}0.2 \\
0 \\
0\end{array}$ & $\begin{array}{l}0 \\
0 \\
0\end{array}$ & $\begin{array}{l}0 \\
0 \\
0\end{array}$ & $\begin{array}{l}0 \\
0 \\
0\end{array}$ \\
\hline$\rho_{2} \widehat{-\mathrm{ADD}}\left(\widetilde{\mathbf{x}}_{n}, \overline{\mathbf{x}}_{n}\right)$ & \begin{tabular}{|c|}
10 \\
30 \\
100 \\
\end{tabular} & $\begin{array}{c}78.2 \\
69 \\
79.1 \\
\end{array}$ & $\begin{array}{l}70.1 \\
52.2 \\
25.7 \\
\end{array}$ & $\begin{array}{c}11.3 \\
1.6 \\
0 \\
\end{array}$ & $\begin{array}{c}6.1 \\
0.1 \\
0 \\
\end{array}$ & $\begin{array}{c}0.2 \\
0 \\
0 \\
\end{array}$ & $\begin{array}{l}0 \\
0 \\
0 \\
\end{array}$ & $\begin{array}{l}0 \\
0 \\
0 \\
\end{array}$ & $\begin{array}{l}0 \\
0 \\
0 \\
\end{array}$ \\
\hline$\widehat{\rho_{1}-\mathrm{ADD}}\left(\widetilde{\mathbf{x}}_{n}, \widehat{\widehat{\mathrm{Me}}}\left(\widetilde{\mathbf{x}}_{n}\right)\right)$ & \begin{tabular}{|l}
10 \\
30 \\
100 \\
\end{tabular} & $\begin{array}{l}79.2 \\
63.5 \\
64.9 \\
\end{array}$ & $\begin{array}{l}76.2 \\
72.3 \\
58.9 \\
\end{array}$ & $\begin{array}{c}14.2 \\
1.9 \\
0 \\
\end{array}$ & $\begin{array}{c}16.7 \\
3.1 \\
0 \\
\end{array}$ & $\begin{array}{c}0.2 \\
0 \\
0 \\
\end{array}$ & $\begin{array}{c}0.1 \\
0 \\
0 \\
\end{array}$ & $\begin{array}{l}0 \\
0 \\
0 \\
\end{array}$ & $\begin{array}{l}0 \\
0 \\
0 \\
\end{array}$ \\
\hline 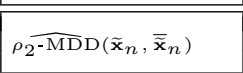 & \begin{tabular}{|l}
10 \\
30 \\
100 \\
\end{tabular} & $\begin{array}{r}91 \\
88.7 \\
94.9 \\
\end{array}$ & $\begin{array}{l}86.3 \\
89.1 \\
95.7 \\
\end{array}$ & $\begin{array}{l}47.9 \\
42.8 \\
55.3 \\
\end{array}$ & $\begin{array}{l}41.1 \\
42.8 \\
55.8 \\
\end{array}$ & $\begin{array}{ll}14.3 \\
8.7 \\
4.3 \\
\end{array}$ & $\begin{array}{l}9.4 \\
7.7 \\
3.7 \\
\end{array}$ & $\begin{array}{c}2.3 \\
0.4 \\
0 \\
\end{array}$ & $\begin{array}{c}0.7 \\
0 \\
0 \\
\end{array}$ \\
\hline 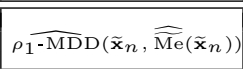 & $\begin{array}{l}10 \\
30 \\
100 \\
\end{array}$ & $\begin{array}{c}93 \\
97.1 \\
100\end{array}$ & $\begin{array}{l}91.2 \\
8.6 \\
74.3\end{array}$ & $\begin{array}{l}66.2 \\
83.5 \\
97.5 \\
\end{array}$ & $\begin{array}{l}57.5 \\
37.8 \\
12.5\end{array}$ & $\begin{array}{l}40.7 \\
47.8 \\
44.7\end{array}$ & $\begin{array}{c}25 \\
9 \\
0.7\end{array}$ & $\begin{array}{c}15.7 \\
11.2 \\
1.2 \\
\end{array}$ & $\begin{array}{l}8.3 \\
3.2 \\
0.1\end{array}$ \\
\hline
\end{tabular}

\begin{tabular}{|c|c|c|c|c|c|c|c|c|c|}
\hline \multirow{3}{*}{$\frac{\widehat{\mathrm{D}}}{\widehat{\rho_{2}-\mathrm{SD}}\left(\widetilde{\mathbf{x}}_{n}\right)}$} & \multirow{3}{*}{$\begin{array}{c}n \\
10 \\
30 \\
100 \\
\end{array}$} & \multicolumn{8}{|c|}{$\begin{array}{c}\%|\widehat{\mathrm{D}}(\mathrm{FRS})-\widehat{\mathrm{D}}(\mathrm{S})|>\varepsilon \quad(k=4, \beta(p, q) \equiv \beta(0.75,0.75)) \\
\end{array}$} \\
\hline & & \multicolumn{2}{|c|}{$\begin{aligned} \varepsilon & =1 \\
\mathrm{~S} & =\mathrm{NEL} \mid \mathrm{S}=\mathrm{FLS}\end{aligned}$} & \multicolumn{2}{|c|}{$\begin{aligned} \varepsilon & =5 \\
\mathrm{~S} & =\mathrm{NEL} \mid \mathrm{S}=\mathrm{FLS}\end{aligned}$} & \multicolumn{2}{|c|}{$\begin{array}{c}\varepsilon=10 \\
S=N E L \mid S=F L S\end{array}$} & \multicolumn{2}{|c|}{$\begin{array}{c}\varepsilon=15 \\
\mathrm{~S}=\mathrm{NEL} \mid \mathrm{S}=\mathrm{FLS}\end{array}$} \\
\hline & & $\begin{array}{c}83 \\
90 \\
98.8\end{array}$ & $\begin{array}{c}67.9 \\
59.7 \\
70\end{array}$ & $\begin{array}{l}19.9 \\
6.2 \\
0.1\end{array}$ & $\begin{array}{c}3.8 \\
0 \\
0\end{array}$ & $\begin{array}{l}0 \\
0 \\
0\end{array}$ & $\begin{array}{l}0 \\
0 \\
0\end{array}$ & $\begin{array}{l}0 \\
0 \\
0\end{array}$ & $\begin{array}{l}0 \\
0 \\
0\end{array}$ \\
\hline$\widehat{\rho-\mathrm{ADD}}\left(\widetilde{\mathbf{x}}_{n}, \overline{\mathbf{x}}_{n}\right)$ & $\begin{array}{c}10 \\
30 \\
100\end{array}$ & $\begin{array}{l}76.4 \\
70.7 \\
82.1\end{array}$ & $\begin{array}{c}71.4 \\
55.4 \\
40\end{array}$ & $\begin{array}{c}12.1 \\
2.2 \\
0\end{array}$ & $\begin{array}{c}5.3 \\
0 \\
0\end{array}$ & $\begin{array}{l}0 \\
0 \\
0\end{array}$ & $\begin{array}{l}0 \\
0 \\
0 \\
0\end{array}$ & $\begin{array}{l}0 \\
0 \\
0\end{array}$ & $\begin{array}{l}0 \\
0 \\
0 \\
0\end{array}$ \\
\hline$\widehat{\rho-\mathrm{ADD}}\left(\widetilde{\mathbf{x}}_{n}, \widehat{\widehat{\mathrm{Me}}}\left(\widetilde{\mathbf{x}}_{n}\right)\right)$ & $\begin{array}{l}10 \\
30 \\
100\end{array}$ & $\begin{array}{c}78 \\
65.9 \\
65.3\end{array}$ & $\begin{array}{l}78.4 \\
77.8 \\
77.6\end{array}$ & $\begin{array}{c}14.1 \\
1.3 \\
0\end{array}$ & $\begin{array}{l}14 \\
2.7 \\
0\end{array}$ & $\begin{array}{ll}0 \\
0 \\
0 \\
0\end{array}$ & $\begin{array}{l} \\
0 \\
0 \\
0\end{array}$ & $\begin{array}{l}0 \\
0 \\
0 \\
0\end{array}$ & $\begin{array}{ll}0 \\
0 \\
0 \\
0\end{array}$ \\
\hline$\rho_{2} \widehat{-\mathrm{MDD}}\left(\tilde{\mathbf{x}}_{n}, \overline{\mathbf{x}}_{n}\right)$ & $\begin{array}{l}10 \\
30 \\
100 \\
\end{array}$ & $\begin{array}{l}87.9 \\
91.5 \\
98.5\end{array}$ & $\begin{array}{c}87.8 \\
92 \\
99.2\end{array}$ & $\begin{array}{l}53.2 \\
59.5 \\
84.8\end{array}$ & $\begin{array}{l}41.9 \\
56.1 \\
86.8\end{array}$ & $\begin{array}{l}17.4 \\
20.4 \\
31.1\end{array}$ & $\begin{array}{c}9.9 \\
15.3 \\
28.6\end{array}$ & $\begin{array}{l}1.3 \\
0.7 \\
1.2\end{array}$ & $\begin{array}{l}0.2 \\
0.3 \\
0.2 \\
0.2\end{array}$ \\
\hline$\rho_{1-\mathrm{MDD}}\left(\widetilde{\mathbf{x}}_{n}, \widehat{\widehat{\mathrm{Me}}}\left(\tilde{\mathbf{x}}_{n}\right)\right)$ & $\begin{array}{l}10 \\
30 \\
100\end{array}$ & $\begin{array}{c}94 \\
96.5 \\
98.8\end{array}$ & $\begin{array}{l}91.1 \\
83.9 \\
78.1\end{array}$ & $\begin{array}{l}68.5 \\
74.7 \\
74.6\end{array}$ & $\begin{array}{l}57.5 \\
34.6 \\
18.6\end{array}$ & $\begin{array}{l}39.7 \\
36.3 \\
13.5\end{array}$ & $\begin{array}{c}22.6 \\
9.5 \\
4.8\end{array}$ & $\begin{array}{l}15.5 \\
5.6 \\
0.3\end{array}$ & $\begin{array}{l}6.1 \\
2.1 \\
0.3\end{array}$ \\
\hline
\end{tabular}

\begin{tabular}{|c|c|c|c|c|c|c|c|c|c|}
\hline \multirow{3}{*}{$\frac{\widehat{\mathrm{D}}}{\widehat{\rho_{2-\mathrm{SD}}\left(\widetilde{\mathbf{x}}_{n}\right)}}$} & \multirow{3}{*}{$\begin{array}{c}n \\
10 \\
30 \\
100\end{array}$} & \multicolumn{8}{|c|}{\begin{tabular}{r|rl}
$\%$ & $\hat{\mathrm{D}}(\mathrm{FRS})-\widehat{\mathrm{D}}(\mathrm{S}) \mid>\varepsilon \quad(k=4, \beta(p, q) \equiv \beta(4,2))$ \\
\end{tabular}} \\
\hline & & \multicolumn{2}{|c|}{$\begin{aligned} \varepsilon & =1 \\
S & =\mathrm{NEL} \mid \mathrm{S}\end{aligned}=\mathrm{FLS}$} & \multicolumn{2}{|c|}{$\begin{aligned} \varepsilon & =5 \\
\mathrm{~S} & =\mathrm{NEL} \mid \mathrm{S}=\mathrm{FLS}\end{aligned}$} & \multicolumn{2}{|c|}{$\begin{array}{c}\varepsilon=10 \\
S=N E L \mid S=F L S\end{array}$} & \multicolumn{2}{|c|}{$\begin{array}{c}\varepsilon=15 \\
\mathrm{~S}=\mathrm{NEL} \mid \mathrm{S}=\mathrm{FLS}\end{array}$} \\
\hline & & $\begin{array}{c}81 \\
81 \\
93.1\end{array}$ & $\begin{array}{l}71.7 \\
50.6 \\
22.3\end{array}$ & $\begin{array}{c}18.9 \\
3.5 \\
0\end{array}$ & $\begin{array}{c}4.9 \\
0 \\
0\end{array}$ & $\begin{array}{c}0.6 \\
0 \\
0\end{array}$ & $\begin{array}{c}0.6 \\
0 \\
0\end{array}$ & $\begin{array}{l}0 \\
0 \\
0\end{array}$ & $\begin{array}{l}0 \\
0 \\
0\end{array}$ \\
\hline 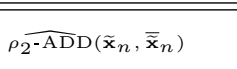 & $\begin{array}{l}10 \\
30 \\
100\end{array}$ & $\begin{array}{c}77.6 \\
70 \\
85.8\end{array}$ & $\begin{array}{l}73.1 \\
75.5 \\
95.9\end{array}$ & $\begin{array}{c}10.3 \\
5 \\
0.6\end{array}$ & $\begin{array}{l}0.3 \\
5.7 \\
0.4\end{array}$ & $\begin{array}{c}0.3 \\
0 \\
0\end{array}$ & $\begin{array}{l}0 \\
0 \\
0\end{array}$ & $\begin{array}{l}0 \\
0 \\
0\end{array}$ & $\begin{array}{l}0 \\
0 \\
0\end{array}$ \\
\hline$\rho_{1-\mathrm{ADD}}\left(\tilde{\mathbf{x}}_{n}, \widehat{\widehat{\mathrm{Me}}}\left(\widetilde{\mathbf{x}}_{n}\right)\right)$ & \begin{tabular}{|c|}
10 \\
30 \\
100 \\
\end{tabular} & $\begin{array}{l}79.2 \\
76.5 \\
92.8 \\
\end{array}$ & $\begin{array}{l}84.2 \\
95.7 \\
100 \\
\end{array}$ & $\begin{array}{l}16 \\
5.6 \\
0.2 \\
0.2\end{array}$ & $\begin{array}{l}20.3 \\
16.4 \\
5.8 \\
\end{array}$ & $\begin{array}{c}0.1 \\
0 \\
0\end{array}$ & $\begin{array}{l}0 \\
0 \\
0\end{array}$ & $\begin{array}{ll}0 \\
0 \\
0 \\
\end{array}$ & $\begin{array}{l}0 \\
0 \\
0 \\
\end{array}$ \\
\hline 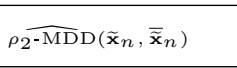 & $\begin{array}{l}10 \\
30 \\
100 \\
\end{array}$ & $\begin{array}{l}92.5 \\
97.1 \\
100 \\
\end{array}$ & $\begin{array}{l}92.8 \\
97.4 \\
100\end{array}$ & $\begin{array}{c}62.4 \\
90.7 \\
100\end{array}$ & $\begin{array}{l}61.9 \\
91.4 \\
100\end{array}$ & $\begin{array}{l}21.7 \\
51.9 \\
86.3\end{array}$ & $\begin{array}{c}13 \\
37 \\
61.7\end{array}$ & $\begin{array}{l}2.6 \\
3.2 \\
1.3 \\
\end{array}$ & $\begin{array}{c}0 \\
0.2 \\
0 \\
\end{array}$ \\
\hline$\rho_{1} \widehat{\widehat{-M D D}}\left(\widetilde{\mathbf{x}}_{n}, \widehat{\widehat{\mathrm{Me}}}\left(\widetilde{\mathbf{x}}_{n}\right)\right)$ & \begin{tabular}{|c|}
10 \\
30 \\
100
\end{tabular} & $\begin{array}{l}96.5 \\
98.1 \\
100\end{array}$ & $\begin{array}{l}96.1 \\
99.3 \\
100\end{array}$ & $\begin{array}{l}84.9 \\
95.4 \\
100\end{array}$ & $\begin{array}{l}80.6 \\
95.4 \\
100\end{array}$ & $\begin{array}{c}42 \\
73.7 \\
96.1\end{array}$ & $\begin{array}{l}36.5 \\
72.4 \\
96.1\end{array}$ & $\begin{array}{l}9.2 \\
7.7 \\
0.7\end{array}$ & $\begin{array}{c}4 \\
5.7 \\
0.7\end{array}$ \\
\hline
\end{tabular}

\begin{tabular}{|c|c|c|c|c|c|c|c|c|c|}
\hline \multirow[b]{2}{*}{$\widehat{\mathrm{D}}$} & \multirow[b]{2}{*}{$n$} & \multicolumn{8}{|c|}{$\%|\hat{\mathrm{D}}(\mathrm{FRS})-\widehat{\mathrm{D}}(\mathrm{S})|>\varepsilon \quad(k=4, \beta(p, q) \equiv \beta(6,1))$} \\
\hline & & $\mathrm{S}=\mathrm{NE}$ & $\stackrel{1}{\mathrm{~S}}=\mathrm{FLS}$ & $\mathrm{S}=\mathrm{NE}$ & $\mathrm{S}_{\mathrm{S}}^{\mathrm{T}}=\mathrm{FLS}$ & $\mathrm{S}=\mathrm{NE}$ & $\begin{array}{l}10 \\
S=F L S\end{array}$ & $\mathrm{~S}=\mathrm{NEI}$ & $\begin{array}{l}15 \\
S=F L S\end{array}$ \\
\hline$\widehat{\rho_{2}-\mathrm{SD}}\left(\tilde{\mathbf{x}}_{n}\right)$ & $\begin{array}{c}10 \\
30 \\
100 \\
\end{array}$ & $\begin{array}{c}95.4 \\
99.5 \\
100\end{array}$ & $\begin{array}{c}78 \\
76.7 \\
90.3\end{array}$ & $\begin{array}{l}44.2 \\
44.9 \\
37.3 \\
\end{array}$ & $\begin{array}{c}8.6 \\
0.4 \\
0\end{array}$ & $\begin{array}{c}0.3 \\
0 \\
0\end{array}$ & $\begin{array}{l}0 \\
0 \\
0 \\
\end{array}$ & $\begin{array}{l}0 \\
0 \\
0\end{array}$ & $\begin{array}{l}0 \\
0 \\
0\end{array}$ \\
\hline$\rho_{2-\mathrm{ADD}}\left(\tilde{\mathbf{x}}_{n}, \overline{\mathbf{x}}_{n}\right)$ & \begin{tabular}{|c|}
10 \\
30 \\
100 \\
\end{tabular} & $\begin{array}{l}92.8 \\
99.2 \\
100 \\
\end{array}$ & $\begin{array}{l}80.6 \\
91.1 \\
99.4 \\
\end{array}$ & $\begin{array}{l}46.9 \\
63.8 \\
78.4 \\
\end{array}$ & $\begin{array}{c}11.1 \\
2.5 \\
0 \\
\end{array}$ & $\begin{array}{c}1.1 \\
0 \\
0 \\
\end{array}$ & $\begin{array}{l}0 \\
0 \\
0 \\
\end{array}$ & $\begin{array}{ll}0 \\
0 \\
0 \\
\end{array}$ & $\begin{array}{l}0 \\
0 \\
0 \\
\end{array}$ \\
\hline$\rho_{1-\mathrm{ADD}}\left(\tilde{\mathbf{x}}_{n}, \widehat{\widehat{\mathrm{Me}}}\left(\widetilde{\mathbf{x}}_{n}\right)\right)$ & \begin{tabular}{|c|}
10 \\
30 \\
100 \\
\end{tabular} & $\begin{array}{l}81.1 \\
86.7 \\
95.2 \\
\end{array}$ & $\begin{array}{l}68.7 \\
52.9 \\
25.9 \\
\end{array}$ & $\begin{array}{c}23.4 \\
20.6 \\
6.4 \\
\end{array}$ & $\begin{array}{c}3.9 \\
0 \\
0 \\
\end{array}$ & $\begin{array}{c}1.5 \\
0 \\
0 \\
\end{array}$ & $\begin{array}{l}0 \\
0 \\
0 \\
\end{array}$ & $\begin{array}{l}0 \\
0 \\
0 \\
\end{array}$ & $\begin{array}{l}0 \\
0 \\
0 \\
\end{array}$ \\
\hline 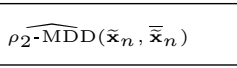 & \begin{tabular}{|c|}
10 \\
30 \\
100 \\
\end{tabular} & $\begin{array}{l}79.4 \\
86.6 \\
98.3 \\
\end{array}$ & $\begin{array}{l}71.9 \\
62.2 \\
60.9 \\
\end{array}$ & $\begin{array}{l}25.2 \\
24.6 \\
12.1 \\
\end{array}$ & $\begin{array}{l}8.4 \\
1.5 \\
0.2\end{array}$ & $\begin{array}{l}2.6 \\
0.1 \\
0.1 \\
\end{array}$ & \begin{tabular}{|c|c|}
0.3 \\
0 \\
0 \\
\end{tabular} & $\begin{array}{c}0.2 \\
0 \\
0\end{array}$ & $\begin{array}{l}0 \\
0 \\
0 \\
0\end{array}$ \\
\hline$\rho_{1} \widehat{-\mathrm{MDD}}\left(\widetilde{\mathbf{x}}_{n}, \widehat{\widehat{\mathrm{Me}}}\left(\widetilde{\mathbf{x}}_{n}\right)\right)$ & \begin{tabular}{|c|}
10 \\
30 \\
100 \\
\end{tabular} & $\begin{array}{l}100 \\
100 \\
100 \\
100\end{array}$ & $\begin{array}{l}98.7 \\
100 \\
100 \\
\end{array}$ & $\begin{array}{l}66.2 \\
88.6 \\
99.1 \\
\end{array}$ & $\begin{array}{l}57.7 \\
85.2 \\
99\end{array}$ & $\begin{array}{l}7.1 \\
2.4 \\
0.1 \\
\end{array}$ & $\begin{array}{l}3.7 \\
2.3 \\
0.1 \\
\end{array}$ & $\begin{array}{l}0.2 \\
0.3 \\
0.1 \\
\end{array}$ & $\begin{array}{c}0 \\
0.2 \\
0.1 \\
0.1\end{array}$ \\
\hline
\end{tabular}


Table 2. \% of simulated samples of size $n$ for which the Euclidean distance between the sample scale estimate $\widehat{D}$ associated with the FRS and the one associated with either the NEL (numerically encoded Likert) or the FLS (fuzzy linguistic scale) with $k=5$ different values is greater than $\varepsilon \in\{1,5,10,15\}$ and (from top to bottom) $\beta(p, q) \equiv \beta(1,1), \beta(.75, .75), \beta(4,2)$, and $\beta(6,1)$

\begin{tabular}{|c|c|c|c|c|c|c|c|c|c|}
\hline \multirow{3}{*}{$\widehat{\widehat{\mathrm{D}}}$} & \multirow{3}{*}{$\begin{array}{c}n \\
10 \\
30 \\
100 \\
\end{array}$} & \multicolumn{8}{|c|}{$\%|\hat{\mathrm{D}}(\mathrm{FRS})-\hat{\mathrm{D}}(\mathrm{S})|>\varepsilon \quad(k=5, \beta(p, q) \equiv \beta(1,1))$} \\
\hline & & \multicolumn{2}{|c|}{$\mathrm{S}=\mathrm{NEL} \mid \stackrel{1}{\mathrm{~S}}=\mathrm{FLS}$} & \multicolumn{2}{|c|}{$\mathrm{S}=\mathrm{NEL}=5$} & \multicolumn{2}{|c|}{$\mathrm{S}=\mathrm{NEL} \mid \mathrm{S}=\mathrm{SLS}$} & \multicolumn{2}{|c|}{$\mathrm{S}=\mathrm{NEL} \mid \mathrm{s}=\mathrm{SLS}$} \\
\hline & & \begin{tabular}{|l|}
71.6 \\
66.2 \\
75.2 \\
\end{tabular} & $\begin{array}{l}58.6 \\
40.5 \\
25.1 \\
\end{array}$ & $\begin{array}{c}4.4 \\
0 \\
0 \\
\end{array}$ & $\begin{array}{c}1.1 \\
0 \\
0 \\
\end{array}$ & \begin{tabular}{|l|}
0 \\
0 \\
0 \\
\end{tabular} & 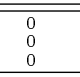 & $\begin{array}{l}0 \\
0 \\
0 \\
\end{array}$ & $\begin{array}{l}\begin{array}{l}0 \\
0 \\
0\end{array} \\
\end{array}$ \\
\hline$\rho_{2-\mathrm{ADD}}\left(\widetilde{\mathbf{x}}_{n}, \overline{\mathbf{x}}_{n}\right)$ & $\begin{array}{c}10 \\
30 \\
100 \\
\end{array}$ & $\begin{array}{l}65.8 \\
48.6 \\
25.1 \\
\end{array}$ & $\begin{array}{l}64.9 \\
60.2 \\
72.7 \\
\end{array}$ & $\begin{array}{c}2.4 \\
0.2 \\
0 \\
\end{array}$ & $\begin{array}{c}1.9 \\
0.2 \\
0 \\
\end{array}$ & $\begin{array}{l}0 \\
0 \\
0 \\
\end{array}$ & $\begin{array}{l}0 \\
0 \\
0 \\
\end{array}$ & $\begin{array}{l}0 \\
0 \\
0 \\
\end{array}$ & $\begin{array}{l}0 \\
0 \\
0 \\
\end{array}$ \\
\hline$\rho_{1-\mathrm{ADD}}\left(\widetilde{\mathbf{x}}_{n}, \widehat{\widehat{\mathrm{Me}}}\left(\widetilde{\mathbf{x}}_{n}\right)\right)$ & $\begin{array}{c}10 \\
30 \\
100 \\
\end{array}$ & $\begin{array}{l}68.3 \\
50.2 \\
20.7 \\
\end{array}$ & $\begin{array}{l}70.4 \\
60.8 \\
75.1 \\
\end{array}$ & $\begin{array}{c}3.7 \\
0.2 \\
0 \\
\end{array}$ & $\begin{array}{c}5.3 \\
0.3 \\
0 \\
\end{array}$ & $\begin{array}{l}0 \\
0 \\
0 \\
\end{array}$ & $\begin{array}{l}0 \\
0 \\
0 \\
\end{array}$ & $\begin{array}{l}0 \\
0 \\
0 \\
\end{array}$ & $\begin{array}{l}0 \\
0 \\
0 \\
\end{array}$ \\
\hline$\rho_{2} \widehat{-\mathrm{MDD}}\left(\widetilde{\mathbf{x}}_{n}, \overline{\mathbf{x}}_{n}\right)$ & $\begin{array}{c}10 \\
30 \\
100 \\
\end{array}$ & $\begin{array}{l}83.9 \\
79.3 \\
71.2 \\
\end{array}$ & $\begin{array}{l}83.2 \\
79.8 \\
70.6 \\
\end{array}$ & $\begin{array}{c}34.3 \\
24.3 \\
7.2 \\
\end{array}$ & $\begin{array}{l}28.4 \\
21.2 \\
6.2 \\
\end{array}$ & $\begin{array}{c}3.6 \\
1.9 \\
0 \\
\end{array}$ & $\begin{array}{c}2.3 \\
0.9 \\
0 \\
\end{array}$ & $\begin{array}{c}0.1 \\
0.1 \\
0 \\
\end{array}$ & $\begin{array}{c}0.1 \\
0.1 \\
0 \\
\end{array}$ \\
\hline$\rho_{1} \widehat{-\mathrm{MDD}}\left(\widetilde{\mathbf{x}}_{n}, \widehat{\widehat{\mathrm{Me}}}\left(\widetilde{\mathbf{x}}_{n}\right)\right)$ & $\begin{array}{c}10 \\
30 \\
100\end{array}$ & $\begin{array}{l}89.7 \\
85.3 \\
73.3 \\
\end{array}$ & $\begin{array}{l}87.3 \\
85.4 \\
73.4 \\
\end{array}$ & $\begin{array}{c}53 \\
33.1 \\
7.3 \\
\end{array}$ & $\begin{array}{c}46.1 \\
29.7 \\
7.2\end{array}$ & $\begin{array}{l}16.7 \\
5.5 \\
0.5\end{array}$ & $\begin{array}{c}13.1 \\
4.6 \\
0.4 \\
\end{array}$ & $\begin{array}{c}2.4 \\
0.3 \\
0 \\
\end{array}$ & $\begin{array}{c}1.9 \\
0.2 \\
0 \\
\end{array}$ \\
\hline
\end{tabular}

\begin{tabular}{|c|c|c|c|c|c|c|c|c|c|}
\hline$\widehat{\mathrm{D}}$ & $n$ & $\mathrm{~S}=\mathrm{NE}$ & \multicolumn{6}{|c|}{ 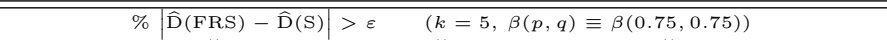 } & $=\mathrm{FLS}$ \\
\hline$\widehat{\rho_{2}-\mathrm{SD}}\left(\widetilde{\mathbf{x}}_{n}\right)$ & $\begin{array}{l}10 \\
30 \\
100 \\
\end{array}$ & $\begin{array}{l}72.8 \\
72.4 \\
83.8\end{array}$ & $\begin{array}{l}64.5 \\
49.1 \\
46.1 \\
\end{array}$ & $\begin{array}{c}3.8 \\
0 \\
0\end{array}$ & $\begin{array}{c}0.8 \\
0 \\
0 \\
\end{array}$ & $\begin{array}{l}0 \\
0 \\
0 \\
0\end{array}$ & $\begin{array}{l}0 \\
0 \\
0\end{array}$ & $\begin{array}{l}0 \\
0 \\
0 \\
\end{array}$ & $\begin{array}{l}0 \\
0 \\
0 \\
0\end{array}$ \\
\hline 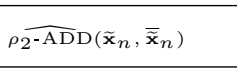 & $\begin{array}{c}10 \\
30 \\
100 \\
\end{array}$ & $\begin{array}{c}68.6 \\
49.3 \\
21 \\
\end{array}$ & $\begin{array}{l}69.4 \\
61.6 \\
78.3 \\
\end{array}$ & $\begin{array}{c}.2 \\
0 \\
0 \\
\end{array}$ & $\begin{array}{c}.2 \\
0 \\
0 \\
\end{array}$ & $\begin{array}{l}0 \\
0 \\
0 \\
\end{array}$ & $\begin{array}{l}0 \\
0 \\
0 \\
\end{array}$ & $\begin{array}{l}0 \\
0 \\
0 \\
0\end{array}$ & $\begin{array}{l}0 \\
0 \\
0 \\
0\end{array}$ \\
\hline$\rho_{1-\mathrm{ADD}}\left(\widetilde{\mathbf{x}}_{n}, \widehat{\widehat{\mathrm{Me}}}\left(\widetilde{\mathbf{x}}_{n}\right)\right)$ & $\begin{array}{l}10 \\
30 \\
100 \\
\end{array}$ & $\begin{array}{l}67.3 \\
50.8 \\
24.4\end{array}$ & $\begin{array}{l}74.2 \\
64.6 \\
79.7\end{array}$ & $\begin{array}{l}4 \\
0 \\
0\end{array}$ & $\begin{array}{c}5.3 \\
0.1 \\
0\end{array}$ & $\begin{array}{l}0 \\
0 \\
0\end{array}$ & $\begin{array}{c}0.1 \\
0 \\
0\end{array}$ & $\begin{array}{l}0 \\
0 \\
0 \\
0\end{array}$ & $\begin{array}{l}0 \\
0 \\
0 \\
0\end{array}$ \\
\hline$\rho_{2-\mathrm{MDD}}\left(\tilde{\mathbf{x}}_{n}, \overline{\mathbf{x}}_{n}\right)$ & \begin{tabular}{|c|c|}
10 \\
30 \\
100
\end{tabular} & $\begin{array}{l}86.4 \\
86.3 \\
79.4\end{array}$ & $\begin{array}{l}83.5 \\
86.8 \\
78.9\end{array}$ & $\begin{array}{l}36.7 \\
33.5 \\
13.7\end{array}$ & $\begin{array}{l}29.8 \\
28.8 \\
12.4\end{array}$ & $\begin{array}{l}4.3 \\
2.5 \\
0.2\end{array}$ & $\begin{array}{c}2.6 \\
1 \\
0.1\end{array}$ & $\begin{array}{l}0 \\
0 \\
0 \\
0\end{array}$ & $\begin{array}{l}0 \\
0 \\
0\end{array}$ \\
\hline$\rho_{1-\widehat{M D D}}\left(\widetilde{\mathbf{x}}_{n}, \widehat{\widehat{\mathrm{Me}}}\left(\widetilde{\mathbf{x}}_{n}\right)\right)$ & \begin{tabular}{|l|l|}
10 \\
30 \\
100
\end{tabular} & $\begin{array}{l}90 \\
85.7 \\
78.3\end{array}$ & $\begin{array}{l}85.1 \\
86.4 \\
79.2\end{array}$ & $\begin{array}{l}52.3 \\
33.9 \\
12.1\end{array}$ & $\begin{array}{l}43.8 \\
32.6 \\
13.4\end{array}$ & $\begin{array}{l}14.1 \\
6.1 \\
0.7\end{array}$ & $\begin{array}{c}10.8 \\
4.8 \\
0.6\end{array}$ & $\begin{array}{c}2 \\
0.2 \\
0\end{array}$ & $\begin{array}{c}1.4 \\
0 \\
0\end{array}$ \\
\hline
\end{tabular}

\begin{tabular}{|c|c|c|c|c|c|c|c|c|c|}
\hline \multirow{3}{*}{$\frac{\widehat{\mathrm{D}}}{\widehat{\rho_{2}-\mathrm{SD}}\left(\widetilde{\mathbf{x}}_{n}\right)}$} & \multirow{3}{*}{$\begin{array}{c}n \\
10 \\
30 \\
100\end{array}$} & \multicolumn{8}{|c|}{$\%|\hat{\mathrm{D}}(\mathrm{FRS})-\hat{\mathrm{D}}(\mathrm{S})|>\varepsilon \quad(k=5, \beta(p, q) \equiv \beta(4,2))$} \\
\hline & & \multicolumn{8}{|c|}{ 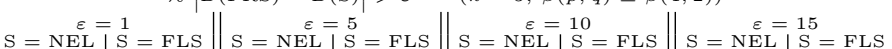 } \\
\hline & & $\begin{array}{l}70.7 \\
57.5 \\
53.5\end{array}$ & $\begin{array}{l}60.7 \\
39.1 \\
12.6\end{array}$ & $\begin{array}{c}4.4 \\
0 \\
0\end{array}$ & $\begin{array}{c}1.2 \\
0 \\
0\end{array}$ & $\begin{array}{l}0 \\
0 \\
0\end{array}$ & $\begin{array}{l} \\
0 \\
0\end{array}$ & $\begin{array}{l}0 \\
0 \\
0\end{array}$ & $\begin{array}{l}0 \\
0 \\
0\end{array}$ \\
\hline 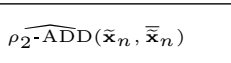 & $\begin{array}{c}10 \\
30 \\
100\end{array}$ & $\begin{array}{l}67.9 \\
48.4 \\
33.1\end{array}$ & $\begin{array}{l}64.4 \\
43.7 \\
19.5\end{array}$ & $\begin{array}{c}3.3 \\
0.2 \\
0\end{array}$ & $\begin{array}{c}2.4 \\
0 \\
0\end{array}$ & $\begin{array}{l}0 \\
0 \\
0\end{array}$ & $\begin{array}{l}0 \\
0 \\
0\end{array}$ & $\begin{array}{l}0 \\
0 \\
0\end{array}$ & $\begin{array}{l}0 \\
0 \\
0\end{array}$ \\
\hline$\rho_{1-\mathrm{ADD}}\left(\widetilde{\mathbf{x}}_{n}, \widehat{\widehat{\mathrm{Me}}}\left(\widetilde{\mathbf{x}}_{n}\right)\right)$ & $\begin{array}{c}10 \\
30 \\
100\end{array}$ & $\begin{array}{c}72.2 \\
58 \\
42.8\end{array}$ & $\begin{array}{l}74.7 \\
66.5 \\
61.4\end{array}$ & $\begin{array}{c}6.7 \\
0.2 \\
0.2\end{array}$ & $\begin{array}{c}7.3 \\
0.5 \\
0\end{array}$ & $\begin{array}{l}0 \\
0 \\
0\end{array}$ & $\begin{array}{l} \\
0 \\
0 \\
0\end{array}$ & $\begin{array}{l}0 \\
0 \\
0\end{array}$ & $\begin{array}{l}0 \\
0 \\
0\end{array}$ \\
\hline$\widehat{\rho_{2}-\overline{M D D}\left(\tilde{\mathbf{x}}_{n}, \overline{\mathbf{x}}_{n}\right)}$ & $\begin{array}{c}10 \\
30 \\
100\end{array}$ & $\begin{array}{l}85.1 \\
82.3 \\
86.5\end{array}$ & $\begin{array}{c}84.7 \\
80 \\
83.3\end{array}$ & $\begin{array}{l}36.8 \\
32.1 \\
29.4\end{array}$ & $\begin{array}{l}31.1 \\
26.2 \\
19.7\end{array}$ & $\begin{array}{l}4.7 \\
2.3 \\
0.3\end{array}$ & $\begin{array}{l}2.1 \\
0.7 \\
0.1\end{array}$ & $\begin{array}{c}0.1 \\
0 \\
0\end{array}$ & $\begin{array}{l}0 \\
0 \\
0\end{array}$ \\
\hline 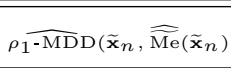 & $\begin{array}{c}10 \\
30 \\
100\end{array}$ & $\begin{array}{l}89.7 \\
92.8 \\
95.3\end{array}$ & $\begin{array}{l}90.4 \\
96.8 \\
100\end{array}$ & $\begin{array}{l}66.8 \\
79.2 \\
92.8\end{array}$ & $\begin{array}{l}62.9 \\
70.5 \\
84.1 \\
\end{array}$ & $\begin{array}{l}23.9 \\
53.1 \\
84.5\end{array}$ & $\begin{array}{l}18.8 \\
35.6 \\
56.3 \\
\end{array}$ & $\begin{array}{l}2.2 \\
2.2 \\
0.2\end{array}$ & $\begin{array}{l}1.4 \\
1.4 \\
0.1\end{array}$ \\
\hline
\end{tabular}

\begin{tabular}{|c|c|c|c|c|c|c|c|c|c|}
\hline \multirow{3}{*}{$\begin{array}{l}\widehat{\mathrm{D}} \\
\widehat{\rho_{2}-\mathrm{SD}}\left(\widetilde{\mathbf{x}}_{n}\right)\end{array}$} & \multirow{3}{*}{$\begin{array}{c}n \\
10 \\
30 \\
100 \\
\end{array}$} & \multicolumn{8}{|c|}{$\%|\hat{\mathrm{D}}(\mathrm{FRS})-\hat{\mathrm{D}}(\mathrm{S})|>\varepsilon \quad(k=5, \beta(p, q) \equiv \beta(6,1))$} \\
\hline & & \multicolumn{2}{|c|}{$\begin{array}{r}\varepsilon=1 \\
\mathrm{~S}=\mathrm{NEL} \mid \mathrm{S}=\mathrm{FLS}\end{array}$} & \multicolumn{2}{|c|}{\begin{tabular}{r|r}
$\varepsilon$ & $=5$ \\
$\mathrm{~S}$ & $=\mathrm{NEL} \mid \mathrm{S}=\mathrm{FLS}$ \\
\end{tabular}} & \multicolumn{2}{|c|}{$\begin{array}{c}\varepsilon=10 \\
\mathrm{~S}=\mathrm{NEL} \\
\mathrm{S}=\mathrm{SLS}\end{array}$} & \multicolumn{2}{|c|}{$\begin{array}{c}\varepsilon=15 \\
\mathrm{~S}=\mathrm{NEL} \\
\mathrm{S}=\mathrm{SLS}\end{array}$} \\
\hline & & $\begin{array}{c}88.5 \\
96.7 \\
100\end{array}$ & $\begin{array}{l}63.3 \\
48.2 \\
39.4\end{array}$ & $\begin{array}{c}13.6 \\
2.3 \\
0\end{array}$ & $\begin{array}{c}0.5 \\
0 \\
0\end{array}$ & $\begin{array}{l}0 \\
0 \\
0\end{array}$ & $\begin{array}{l}0 \\
0 \\
0\end{array}$ & $\begin{array}{l}0 \\
0 \\
0 \\
\end{array}$ & $\begin{array}{l}0 \\
0 \\
0\end{array}$ \\
\hline$\widehat{\rho-\mathrm{ADD}}\left(\tilde{\mathbf{x}}_{n}, \overline{\mathbf{x}}_{n}\right)$ & $\begin{array}{c}10 \\
30 \\
100\end{array}$ & $\begin{array}{l}91.1 \\
99.1 \\
100\end{array}$ & $\begin{array}{l}66.3 \\
69.5 \\
86.7\end{array}$ & $\begin{array}{c}16.8 \\
9.5 \\
1.5\end{array}$ & $\begin{array}{c}0.7 \\
0 \\
0 \\
\end{array}$ & $\begin{array}{ll}0 \\
0 \\
0 \\
0\end{array}$ & $\begin{array}{ll}0 \\
0 \\
0\end{array}$ & $\begin{array}{l}0 \\
0 \\
0\end{array}$ & $\begin{array}{l}0 \\
0 \\
0\end{array}$ \\
\hline$\rho_{1-\mathrm{ADD}}\left(\widetilde{\mathbf{x}}_{n}, \widehat{\widehat{\mathrm{Me}}}\left(\widetilde{\mathbf{x}}_{n}\right)\right)$ & $\begin{array}{c}10 \\
30 \\
100\end{array}$ & $\begin{array}{l}81.2 \\
92.6 \\
99.8\end{array}$ & $\begin{array}{l}59.2 \\
44.3 \\
48.1\end{array}$ & $\begin{array}{l}15.4 \\
9.6 \\
5.9\end{array}$ & $\begin{array}{c}0.7 \\
0 \\
0\end{array}$ & $\begin{array}{l}0 \\
0 \\
0 \\
0\end{array}$ & $\begin{array}{l}0 \\
0 \\
0 \\
0\end{array}$ & $\begin{array}{l}0 \\
0 \\
0 \\
0\end{array}$ & $\begin{array}{l}0 \\
0 \\
0 \\
0\end{array}$ \\
\hline 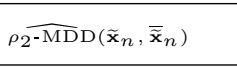 & $\begin{array}{c}10 \\
30 \\
100 \\
\end{array}$ & $\begin{array}{l}83.4 \\
90.3 \\
98.7 \\
\end{array}$ & $\begin{array}{l}69.7 \\
72.5 \\
89.5 \\
\end{array}$ & $\begin{array}{c}22.2 \\
24.5 \\
34 \\
\end{array}$ & $\begin{array}{l}9.2 \\
3.8 \\
0.5 \\
\end{array}$ & $\begin{array}{c}1 \\
0.5 \\
0\end{array}$ & $\begin{array}{l}0 \\
0 \\
0 \\
\end{array}$ & $\begin{array}{l}0 \\
0 \\
0 \\
0\end{array}$ & $\begin{array}{l}0 \\
0 \\
0 \\
\end{array}$ \\
\hline 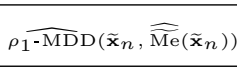 & $\begin{array}{c}10 \\
30 \\
100 \\
\end{array}$ & $\begin{array}{l}99.1 \\
100 \\
100 \\
\end{array}$ & $\begin{array}{l}93.4 \\
96.5 \\
99.5\end{array}$ & $\begin{array}{l}57.4 \\
79.2 \\
96\end{array}$ & $\begin{array}{c}40 \\
66.7 \\
89.7\end{array}$ & $\begin{array}{c}5.4 \\
11.6 \\
21 \\
\end{array}$ & $\begin{array}{c}2.9 \\
8.2 \\
19.9 \\
\end{array}$ & $\begin{array}{l}1.5 \\
9.7 \\
21 \\
\end{array}$ & $\begin{array}{l} \\
0 \\
0 \\
0\end{array}$ \\
\hline
\end{tabular}


The percentages have been quantified over 1000 samples of $n \in\{10,30,100\}$ FRS simulated (with different betas) data with reference interval $[0,100]$ (this last fact being irrelevant for the study). On the basis of Tables 1 and 2 we cannot get very general conclusions, but we can definitely assert that scale measures mostly vary more from the FRS-based data to the encoded Likert ones.

Furthermore, one can state some approximate behaviour patterns, such as

- for almost all situations, the robust scale estimate (the last one) provides us with much higher percentages than non-robust ones; more concretely, $\widehat{\rho_{1}-\mathrm{MDD}}\left(\widetilde{\mathbf{x}}_{n}, \widehat{\mathrm{Me}}\left(\widetilde{\mathbf{x}}_{n}\right)\right)$ is almost generally more sensitive to the change in the rating scale type; this is especially clear for small samples;

- distances are uniformly lower for $k=5$ than for $k=4$ when the midpoint of the 1-level is beta distributed with $(p, q) \in\{(1,1),(0.75,0.75),(4,2)\}$; when $(p, q)=(6,1)$ such a conclusion is appropriate for robust estimates and $\varepsilon \in\{1,5\}$, but there is no clear conclusion for non-robust estimates or greater values of $\varepsilon$.

Acknowledgements The research is this paper has been partially supported by the Spanish Ministry of Economy, Industry and Competitiveness Grant MTM2015-63971P. Its support is gratefully acknowledged.

\section{References}

1. Diamond, P., Kloeden, P.: Metric spaces of fuzzy sets. Fuzzy Sets Syst., 35, 241-249 (1990)

2. De la Rosa de Sáa, S., Lubiano, S., Sinova, S., Filzmoser, P.: Robust scale estimators for fuzzy data. Adv. Data Anal. Classif. 11, 731-758 (2017)

3. Gil, M.A., Lubiano, M.A., De la Rosa de Sáa, S., Sinova, B.: Analyzing data from a fuzzy rating scale-based questionnaire. A case study. Psicothema 27, 182-191 (2015)

4. Herrera, F., Herrera-Viedma, E., Martínez, L.: A fuzzy linguistic methodology to deal with unbalanced linguistic term sets. IEEE T. Fuzzy Syst. 16 (2), 354-370 (2008)

5. Hesket, T., Pryor, R., Hesketh, B.: An application of a computerized fuzzy graphic rating scale to the psychological measurement of individual differences. Int. J. ManMach. Stud. 29, 21-35 (1988)

6. Lubiano, M.A., De la Rosa de Sáa, S., Montenegro, M., Sinova, B., Gil, M.A.: Descriptive analysis of responses to items in questionnaires. Why not using a fuzzy rating scale? Inf. Sci. 360, 131-148 (2016)

7. Lubiano, M.A., Montenegro, M., Sinova, B., De la Rosa de Sáa, S., Gil, M.A.: Hypothesis testing for means in connection with fuzzy rating scale-based data: algorithms and applications. Eur. J. Oper. Res. 251, 918-929 (2016)

8. Lubiano, M.A., Salas, A., Gil, M.A.: A hypothesis testing-based discussion on the sensitivity of means of fuzzy data with respect to data shape. Fuzzy Sets Syst. 328, 54-69 (2017)

9. Puri, M.L., Ralescu, D.A.: Fuzzy random variables. J. Math. Anal. Appl. 114, 409422 (1986)

10. Sinova, B., Gil, M.A., Colubi, A., Van Aelst, S. The median of a random fuzzy number. The 1-norm distance approach. Fuzzy Sets Syst. 200, 99-115 (2012) 